\title{
A Language and Culture Comparative Study of Three Primary Colors in English and Chinese Idioms and Usual Expressions from the Perspective of Metaphorical Cognition
}

\author{
$\mathrm{Pu}$ Meng $^{1 *}$ \\ ${ }^{1}$ School of Western Languages, Harbin Normal University, Harbin, Hei Longjiang, China \\ *Corresponding author. Email: guanghua.ren@gecacademy.cn
}

\begin{abstract}
Color terms, especially the three primary colors, bear the weight of basic meaning of color in English and Chinese idioms and usual expressions. The same color has different meanings in different languages, thus it makes Native English speakers and Native Chinese speakers who use other foreign languages puzzled. Therefore, it is necessary to make a language and culture comparative study of the meanings of three primary colors in English and Chinese idioms and usual expressions. This essay uses metaphors and cognition to make the comparative study between color idioms and usual expressions in English and Chinese. It aims at revealing the similarities and dissimilarities of metaphorical cognitive meanings of three primary colors in English and Chinese idioms and usual expressions. This essay provides a perspective for the role of cultural variation of metaphor and plays a role in overcoming the language barriers in communication.
\end{abstract}

Keywords: three primary colors, idioms and usual expressions, metaphorical cognition.

\section{INTRODUCTION}

Color features are the perception and description of human language. It is an important part of the world language. In language, this symbol symbolizes the an understanding of color words. The words of color are generally used in people's cognition and description of the world [1].

Therefore, it is necessary to inherit the colors in human language. Everything in nature, including human language, is in a state of change. The development of language cannot be separated from the development of human language. A more developed language may affect the less developed one. At the same time, it is also conducive to the development of human language in the process of development. In the process of language learning, learners' attention is becoming more and more concentrated[1]. In non-human languages, Kay and McDaniel find an interesting phenomenon, metaphor. The metaphors of color and color terms are closely related. In exploring metaphors and its working mechanisms, the color terms are very useful.
In this essay, metaphorical cognitive meanings of three primary colors in English and Chinese idioms and usual expressions will be studied. Similarities and dissimilarities of three primary colors in English and Chinese idioms and usual expressions will also be searched from the aspect of metaphorical cognition.

This essay is divided into several parts. In the first part, a brief introduction to metaphorical cognition will be shown. In the next part, first it is about similarities of metaphorical cognitive meanings of three primary colors in English and Chinese idioms and usual expressions.Secondly, it is about dissimilarities of metaphorical cognitive meanings of three primary colors in English and Chinese idioms and usual expressions. The last is about the reasons for similarities and dissimilarities of three primary colors' metaphorical cognitive meanings in English and Chinese idioms and usual expressions. The final part is the conclusion.

\section{LITERATURE REVIEW}

Color terms are as common as linguistic phenomena in many kinds of cultures and languages. It is necessary to introduce color terms, as color terms are going to be 
analyzed and studied in this thesis. In this paper, the author summarizes the former researches from the points of home and abroad.

\subsection{Foreign Studies on Color Terms}

In western countries, the study of color terms has a really long history. Nearly 2500 years ago, Plato made the first exploration of color. His reason was "repeated with only slight variation for 2500 years and carried into the physical sciences as a basic assumption about the nature of color"[2]. He explained that "color" refers to the two colors of "black" and "white". The turn of the century challenges the cross-cultural communication of cross century national civilization. The language is developed with other colors, such as "green" and "yellow".

Until twenty years later, the researchers lead to the theoretical controversy of the terms of color and the revolutionary characteristics of the primitives in the naming. It is the stage of developing color terms, and it is also the industrialization of people's sense of color. Scholars made a cross cultural research and methodological study of intercultural vocabulary in 1960s. And this research is made up of an experimental research institute, including the mother tongue sermons in the twentieth century language background and a survey of seventy or eighty languages. This paper is claiming that "a total universal inventory of eleven basic color categories"[1]. In 1977, the experiments began. Rosch said "color categories are processed by the human mind in terms of their internal structure"[3]. Until now, there are researchers working for color terms in many perspectives.

All in all, about foreign studies on color terms, this thesis showed it timely. The foreign study of color terms developed from "black" and "white" to many kinds of colors, from only English to cross-cultural color terms. Although the color term developed a lot, it also has many drawbacks, for example, the former researches did not pay much attention to the meaning connections of the color terms. This thesis will pay attention to the meaning connections of the color terms in English and Chinese.

\subsection{Domestic Studies on Color Terms}

With the economic development, China's automobile market is expanding and the demand for cars from Chinese consumers is also growing. In recent years, many domestic experts and scholars have studied the Chinese translation of car brand names.

In China, experts and scholars who study foreign languages have also made a great number of researches about color terms. But it started relatively later. Since the 1970s, with the development of sociolinguistics, the attention of people to sociolinguistics has attracted more and more attention to the special functions of color words. We have found several Chinese researches work on color terms. In his book Ying Yu Sheng Se Ci Yu Fan Yi, Zhang Peiji gave a detailed description of all the English terms. And the color terms' translations are described by him. And then in 1990, Liu Yunquan published his book Yu Yan De Yan Se Mei. In this book, he has made a comprehensive accounting of color terms' strategies and functions in China. The color term and metaphor is connecting. In the west, the notions of metaphor depend on specific registers of similarity and dissimilarity. And this is according to David Punter. While in Chinese culture, the role of metaphor is absolutely different[4]. The metaphor's study had the progress since Song Dynasty. Until the publication to Introduction to Rhetoric by Chen Wangdao, a new era of metaphor study appeared [5].

In modern times, we find most of the study is still in rhetoric field and comparison of different tropes. Paper and books on metaphor are rare to see in China. Yet fortunately, researches on metaphor become flourish in China in recent years, and metaphor becomes interesting in various disciplines. Some books have published about metaphor, such as Shu Dingfang's Studies in Metaphor in 2000, Hu Zhuanglin's Metaphor and Cognition in 2004. Chinese scholars also began to pay attention to Chinese metaphor from new perspectives, above the color terms. Domestic studies on color terms also have a great number. And it is developed from foreign studies, and developed the studies of similarity and dissimilarity between English and Chinese. Then the color term study connected with metaphor. The domestic studies developed very quickly, while it has the problem. The problem was that the researches on color term and the researches on metaphor were separated in some periods of time. This thesis analyses red, yellow and blue from the perspective of metaphorical cognition.

\section{A BRIRF INTRODUCTION TO METAPHORICAL CONGNITION}

Here the essay makes a brief introduction to metaphorical cognition. It will introduce the definition of metaphor. And then, it will talk about the introduction to cognition. The relationship between cognitive metaphors will be briefly studied at the end.

Metaphor includes the relationship between two concepts. They are the target domain and source domain. The metaphor describes the target group. And the source domain describes the experience. Classical views regard metaphors as a figure of speech and this kind of speech using one thing to refer to another. After many years of research, the contemporary theory of metaphor came up with the publication of Lakoff and Johnson's Metaphors We Live By in 1980. This book points out that the study of metaphor has developed into 
another stage which shifted from the ornamental function of metaphor to the other functions of it. "Metaphor involves the comparison of two concepts in that one is construed in terms of the other. It is often described in terms of the target domain and the source domain. The target domain is the experience being described by the metaphor and the source domain means that we use in order to describe the experience." [5]. Therefore, metaphors have a close connection with the shaping of the human cognitive system. And metaphors are reflected in the language we use every day.

The scientific research of mind and intelligence is commonly defined as Cognition. Cognitive science is a multidisciplinary study. In the study, the science of cognitive is above psychology, linguistics, and so on. Cognitive linguistics is the basis for learning the language of the world. It began in the 1970s and has been more prosperous since the 1980s. George Lakoff made the further establishment of cognitive linguistics. After that, relevant associations about cognitive linguistics were established. "Linguistic structure depends on conceptualization, the latter being conditioned by our experiential view holds that since our everyday language, it can thus be gleaned from the way we express our ideas."[6]. These languages can be grouped together, because they depend on the perception and classification of the world language. Metaphor research gradually shifts to the study of cognitive metaphors. This is an important part of metaphorical language reflecting human thinking. In Lakoff's view, metaphor pervades in many systems, and "our ordinary conceptual system in terms of which we both think and act, and it is fundamentally metaphorical in nature"[7]. This reflects the expression of daily use.

In summary, cognition and metaphor both developed are still being studied now. Metaphor research is earlier than cognitive metaphors. And the latter was developed from the former. Thus, they are closely related.

\section{BRIRF METAPHORICAL CONGNITION ANALYSIS OF THREE PRIMARY COLORS IN ENGLISH AND CHINESE IDIOMS AND USUAL EXPRESSIONS}

\subsection{Similarities of Metaphorical Cognitive Meanings of Three Primary Colors in English and Chinese Idioms and Usual Expressions}

In this chapter, the analysis of the three pairs of metaphorical color terms in English and Chinese: “red”and “红”, “yellow”and “黄”, “blue”and “蓝”"will be discussed. In the first part, the metaphorical meaning of color and semantics is given. In the second part, different metaphorical meanings of color terms and reasons will be shown.

\section{Similarities: Red}

The similarities of metaphorical cognitive meanings of red will be analyzed with a few typical examples. From the perspective of metaphors, "red" is one of the most vivid colors. The wedding is known as “红事” in Chinese, while in English, "red-letter days" refers to festivals. "In the red”, or “赤字” means the deficit, or more money. "Red" means danger in English. Those people are suffering from disease or catastrophic disasters can get help from the "Red Cross". "Red alter" and “红色警报” are about sudden dangers. “Like a red flag to a bull” and “我从没和人红过脸” mean somebody has never been angry with the other. "Red" and "红" also mean the emotion of embarrassment. In two languages, "to become red-faced" and "脸红” both mean embarrassment.

\section{Similarities: Yellow}

The similarities of metaphorical cognitive meanings of yellow idioms and usual expressions in English and Chinese will be analyzed with some special examples. In the field of metaphorical cognition, "yellow" means many things, such as money, power, and so on. If an athlete makes a mistake a football referee will show a “yellow card” or “黄牌”. Later, it was replaced by all the countries of the world as a symbol. In addition, "yellow" is gold and sun, and it is a symbol of wealth and power. It is used to describe doing important things, like opening ceremonies, because “黄道吉日” is a holy day in Chinese. And in the western countries, "yellow" is the holy color of Christian.

\section{Similarities: Blue}

The similarities of metaphorical cognitive meanings of blue in English and Chinese idioms and usual expressions will be analyzed with several special examples. In people's cognition, "blue" is the color of the sky. It can be metaphorized into a job position "blue collar" and “蓝领”. And "blue print" and “蓝图” refer to a plan for a building or something. Additionally, in two languages, "blue" means unexpected things. For example, in Chinese, people use “青天霹雼” to describe suddenly happening things. "Out of the blue" indicates the unexpected things.

In conclusion, there are many similarities of metaphorical cognitive meanings of red, yellow and blue in English and Chinese idioms and usual expressions. When using these two languages, people need to pay attention to the metaphorical cognitive meanings of color terms. 


\subsection{Dissimilarities of Metaphorical Cognitive Meanings of Three Primary Colors in English and Chinese Idioms and Usual Expressions}

The dissimilarities of metaphorical cognitive meanings of red in English and Chinese idioms and usual expressions will be displayed with several typical examples.

\section{Dissimilarities: Red}

Du Fu's “朱门酒肉臭, 路有冻死骨” explaining that “红” means wealth and high position. “朱门” is the painted door which indicates the senior official and noble residence. There are some expressions with "红” with the meaning of fortune and wealth, for example, " 红利”, “分红” and “红包”. In China, “红” is associated with girls. “红粉佳人” can describe pretty girls. And there are many other words with "红" with the same function. "Pink" is an abbreviation for English girls. Moreover, "red" is enviable, such as “眼红”. Red can describe a welcome and popular person, like “红人”. Chinese is difficult to understand some usual expressions with the word "red". Both of "to see red" and "waving a red flag" mean anger. But it is difficult to be understand Chinese people. The first one suddenly means angry, while the second one represents making mistakes that make others mad.

\section{Dissimilarities: Yellow}

The dissimilarities of metaphorical cognitive meanings of yellow in English and Chinese idioms and usual expressions will be shown with some special examples. "Yellow" also has special significance "jealous" in English, because Judas who was a disciple and one of the original Twelve Apostles of Jesus Christ wore the yellow dress. Thus, "yellow with envy" means human skin becomes yellow. "Yellow" is also for cowards. "Be yellow" can be described as a cowardly person. And "A Yellow Dog" has the same function. One can find a big thick book called in nearly all of the American families. In China, there is “黄色书”. While “Yellow Pages” and “黄色书” have totally different meanings. “黄色书” is a "blue book" for English speaking people. There are so many different ways of expressions with “黄” relate to the emperor in Chinese metaphorical cognition. “黄袍” and “皇宫” are imperial dress and the palace. "Purple” is related to the emperor in English. More importantly, inexperienced people can be described as"yellow", such as “黄毛Y头”. What's more, like “黄色书刊”, it can also be pornographic.

\section{Dissimilarities: Blue}

The dissimilarities of metaphorical cognitive meanings of blue in English and Chinese idioms and usual expressions will be studied with some typical examples. In the scope of metaphor, "blue" has the usual cognition. "Blue room" means the reception room in the White House. It is not a room with blue walls. A man who was recognized by the authority is called "A blue-eyed boy". In "make somebody blue", "Blue" refers to "sadness". While there is no meaning in Chinese. What's more, the word "Blue" means "yellow" in Chinese, such as "blue jokes" and so on.

In summary, there are many dissimilarities of metaphorical cognitive meanings of red, yellow and blue in English and Chinese idioms and usual expressions. Therefore, people can reduce language use and understanding mistakes.

\section{CAUSES OF SIMILARITIES}

\subsection{Perceptual Basis}

For human cognition, color vision is an important tool. The extended meaning of similar color terms is derived from the point of human physiology in these two languages. In our bodily experience, concepts are growing. It also explains the structure of imagination, and the meaning of metaphor is the category of representation of experience. With a physical basis, bodily experience structures our conceptual systems. Ancient Chinese people could receive these things and their existence. Through the description of human physiological structure, the world carries out simple color metaphors.

\subsection{Cultural Basis}

Without the perceptual foundation, color metaphor also has a cultural foundation. Lakoff and Johnson made a studied about that. "In other words, what we call 'direct physical experience' is never merely a matter of having a body of a certain sort; rather, every experience takes place within a vast background of cultural presuppositions."'[7]. So people cannot be pay too much attention to the importance of culture of human cognition. This can be expressed in color metaphors in English and Chinese. In a word, there are two main causes of similarities of the metaphorical cognitive meanings of color terms. They are a perceptual basis and cultural basis. With the same perceptual and cultural, some color terms' metaphorical cognitive meanings are the same in English and Chinese.

\section{CAUSES OF DISSIMILARITIES}

\subsection{Nature and Physical Environment}

Languages build up on the basis of natural and physical environment. It is vocabulary which also makes metaphors which are useful in our usual expressions. "Given a certain kind of habitat, speakers 
living there will be attuned (most subconsciously) to bring and phenomena for the metaphorical comprehension and creation of their conceptual universe."[7]. This suggestion is to study the English and Chinese completely different metaphors with the color terms "yellow" and "blue". "Yellow" is the color of earth in the five elements in Chinese. And it is the centre of the five directions. So 'yellow' is chosen as the color of emperor and the imperial family because it's a symbol of territory and power, and ordinary people were forbidden to be dressed in 'yellow' since the beginning of the Tang Dynasty"[8]. As people known that the UK is an entire inland country. Therefore, they cannot use "yellow" as a social interaction in Chinese. Their subsistence areas are closely linked to the sea. Blue is a familiar and favorite color to British People, because it is the color of the sea.

\subsection{Broader Cultural Context}

Traditions, culture and religion will be considered in the following sections. About traditions and customers, people think that both of them have totally different meanings. For example, "Red" has always been special to Chinese. Chinese people like to wear red clothes during Spring Festival. However, the color terms have totally different cultural backgrounds. The reason is people use the color of the wolf may deviate from its color direction. For example, “红茶” and "black tea” have the same meaning with different color words. Lastly, it is religion. Our understanding of the original culture of the world is achieved through various channels. The study of religion provides people with psychological needs.

To summarize, the major causes of dissimilarities of metaphorical cognitive meanings of color terms are the physical environment and the broader cultural context. Environment can change language expression and culture also.

\section{CONCLUSION}

This essay is dedicated to making the comparative study of red, yellow and blue in English and Chinese idioms and usual expressions based on metaphorical cognition. From the research, people can find that there are some similarities and dissimilarities between English and Chinese idioms and usual expressions which are about color terms. For finding the solutions of the language using problems, the essay uses the theory of metaphorical cognition to find the same and different points of English and Chinese usual expressions with the color term. In vocabulary, color terms especially the three primary colors are the special words. The purpose of this paper is to make a contrastive analysis of three basic languages from two aspects, namely, the semantic features and the language expressions of the three basic colors. However, this essay is limited in collecting English and Chinese color terms in the response time.

\section{REFERENCES}

[1] Berlin, B. \& P, Kay. Basic Color Terms: Their University and Evolution. Berkeley: University of California Press, 1969.

[2] Sloane, Patricia. Primary Sources: Selected Writings on Color from Aristotle to Albers. New York: Design Press, 1991.

[3] Rosch, Eleanor. Cognitive representations of semantic categories. Journal of Experimental Psychology, 2005, 4 (2): 91-111.

[4] Punter, D. Metaphor. London \& New York: Routledge, 2007.

[5] $\mathrm{Hu}$ Zhuanglin. Cognitive Metaphor. Beijing: University of Beijing Press, 2004.

[6] Ungerer F. \& Schmid H. J. An Introduction to Cognitive Linguistics. Beijing: Foreign Language Teaching and Research Press, 2001.

[7] Lakoff, G. \& M. Johnson. Metaphors We Live by. Chicago: University of Chic Press, 1980.

[8] Chang Jingyu. Chinese Vocabulary and Culture . Beijing: University of Beijing Press, 1995.

[9] Wierzbicka A. English: Meaning and culture. Beijing: Foreign Language Teaching and Research Press, 2006.

[10] Kovecses, Z. Metaphor in Culture. Cambridge: Cambridge University Press, 2005.

[11] Marina Pakova. The Extent of the Literal Metaphor, polysemy and Theories of concepts. Peking: Peking University Press, 2004.

[12] Lynne, C. \& G. Low. Researching and Applying Metaphor. Cambridge: Cambridge University Press, 1999.

[13] Langacker Ronald W. Foundation of Cognitive Grammar; Vol.I: Theoretical Prerequisites. Stanford: Stanford University, 1987. 